\title{
HOXA11-AS Regulates NIPAL3 via Hsa-miRNA-19a- 3p, Hsa-miR-141-3p and Hsa-miR-140-5p in Keloid Fibroblast: Bioinformatic Analysis and In Vitro Validation
}

\section{Qiang Wang}

Jilin University Second Hospital

Wei Wang

Jilin University First Hospital

Xiaojie Sun ( $\nabla$ sunxj@jlu.edu.cn )

China-Japan Union Hospital of Jilin University https://orcid.org/0000-0001-8253-6359

\section{Research article}

Keywords: HOXA11-AS, NIPAL3, hsa-miRNA-19a-3p, hsa-miR-141-3p, hsa-miR-140-5p, keloid fibroblast, molecular regulation network

Posted Date: August 30th, 2021

DOI: https://doi.org/10.21203/rs.3.rs-823716/v1

License: (c) (1) This work is licensed under a Creative Commons Attribution 4.0 International License.

Read Full License 


\section{Abstract}

Background: The long noncoding RNA HOXA11-AS is significantly increased in keloids with an unknown mechanism.

Methods: Keloid-derived fibroblasts were primarily cultured from keloid explants (Sample 0). Small inference RNAs (siRNAs) against HOXA11-AS were transinfected in two keloid fibroblasts (Sample 1 and Sample 2). Nonspecific siRNA was transinfected into one keloid fibroblast (Sample 3). The blank plasmid was transinfected into one keloid fibroblast (Sample 4). Five keloid fibroblast samples were sequenced for IncRNAs, mRNAs and miRNAs. Differentially expressed (DE) mRNAs or IncRNAs were obtained in HOXA11-AS-knockdown keloid fibroblasts via DE RNAs from sample 1 intersected from sample 2, removing overlapping nonspecific interfered DE RNAs from sample 3 combined with sample 4. Using stepwise bioinformatics, dominantly functioning mRNAs downstream of HOXA11-AS were scored based on a first-step IncRNA-mRNA-protein (LMP) network in keloid fibroblasts, removing overlapping mRNAs from the nonspecific group. Screened genes were further validated by real-time PCR and western blotting. Validated mRNAs were overtaken to predict a sequence-matched competing endogenous ceRNA network involving the HOXA11-AS (down)- miRNA (up)- validated mRNA (down) pathway in keloid fibroblasts.

Results: Six DE profiles (IncRNA, mRNA, miRNA) were obtained in the HOXA11-AS-knockdown group or in the nonspecific interference group. Fourteen dominantly functional genes were enriched in three biological pathways. SNED1, NIPAL3 and VTN were validated in keloid fibroblasts by knocking down HOXA11-AS via real-time PCR. Only NIPAL3 was predicted as a competing endogenous gene downstream of the HOXA11-AS pathway via three sponging miRNAs (hsa-miRNA-19a-3p, hsa-miR-141-3p, hsa-miR$140-5 p)$.

Conclusions: HOXA11-AS regulated NIPAL3 via three miRNAs in keloid fibroblasts.

\section{Background}

Keloids are aberrantly proliferative benign tumors with remarkedly excessive deposition of fibroblasts and extracellular matrix (ECM) extending beyond the normal boundaries. Patients with genetic predisposition developed keloids during wound healing. The molecular pathways of how keloid fibroblasts proliferate into benign keloids remain largely unclear (1). Previous high-throughput profiling has demonstrated that both encoding messenger RNA (mRNA) and long noncoding RNA (IncRNA) as well as microRNA (miRNA) cooperatively participate in the formation of keloids $(2-6)$.

Long noncoding RNA of HOXA11 antisense RNA (HOXA11-AS) was shown to be markedly changed by in vivo array detection in keloids (7-10). Additionally, using in vivo measurements and bioinformatics analysis, numerous microRNAs were shown to be endogenous competing molecular sponges downstream of the HOXA11-AS pathway in keloids (7-9). Notably, all these miRNAs in the HOXA11-AS pathways were heterogeneous, indicating a complicated molecular network. Moreover, HOXA11-AS was also shown to play a substantial role in various tumors(11). Interestingly, it could act as either a tumor 
promoter or tumor suppressor, depending on the type of cancer (reviewed by (11)). Moreover, even in one type of cancer (ovarian cancer), HOXA11-AS was found to be a tumor accelerator(12) or a tumor suppressor(13). All these studies indicated that HOXA11-AS regulated tumor cellular biology both extensively and intensively with a complicated molecular network (1). Therefore, HOXA11-AS might play as an essential "mediator" on benign keloids characterized by "paradoxical" hyperproliferation and high differentiation(14). In the present study, we used primary cultured keloid fibroblasts to investigate in vitro how HOXA11-AS regulates its target genes by a molecular network.

\section{Methods}

\section{Clinical characteristics of patients undergoing complete keloid excision}

Three patients affected with keloids ( 1 male patient, keloid on chest; 2 female patients, both keloid on respective earlaps) underwent keloid excision surgery in October 2019 in the Department of Plastic and Reconstruction Surgery, China-Japan Union Hospital of Jilin University. Keloid explants were collected from the patients under aseptic conditions. The utilization of these removed samples from surgery was approved by the Medical Ethics Committee of China-Japan Union Friendship Hospital of Jilin University, Changchun, China. All patients were informed with written consent. The study was conducted in accordance with the Declaration of Helsinki Principles.

\section{Harvest Keloid-derived Fibroblasts From Keloid Explant}

Fresh excised keloid samples were deeply punched into a 1 Mimi meter cube to obtain multiple keloid mini explants. Each mini-keloid explant was immediately fragmented into tiny pieces for primary culture of keloid-derived fibroblasts based on a previous approach (15). During primary culturing, fresh tiny keloid fragments were washed with phosphate-buffered saline (PBS; Cultilab, SP, Brazil), penicillin (100 $\mathrm{Ul} / \mathrm{ml}$; Gibco, Carlsbad, CA, USA) and streptomycin (100 $\mu \mathrm{m} / \mathrm{ml}$; Gibco) and incubated in Dulbecco's modified Eagle's medium (DMEM; Cultilab) for surface adherence. Consistent culture was conducted in DMEM 15\% fetal bovine serum (FBS; Cultilab), penicillin (100 Ul/ml; Gibco) and streptomycin (100 $\mathrm{\mu g} / \mathrm{ml}$; Gibco) at stable pH under a $5 \% \mathrm{CO} 2$ atmosphere at $37^{\circ} \mathrm{C}$. Keloid-derived fibroblasts were harvested from successfully cultured keloid mini explants for siRNA transfection.

HOXA11-AS interference or nonspecific interference in keloid fibroblasts via small interfering RNA (siRNA)

Strains at passages $2-3$ of primarily cultured keloid fibroblasts were used for siRNA interference using Transfection. The sequence of human IncRNA HOXA11-AS was

TCCACAGCCTTTGCAGGCGGAATATCGGAATAAAGTGGGTCCAGGC. All siRNAs were designed and offered as commercial products from Sigma-Aldrich (Shanghai, China). Three $100 \mathrm{nM}$ siRNA plasmids targeting HOXA11-AS were simultaneously transfected into two keloid fibroblasts (sample 1 and sample 2). 
Simultaneously, plasmids harboring scrambled nonspecific RNAs were transinfected using the same reagents and protocol (sample 3). Three $100 \mathrm{nM}$ nonspecific interfered siRNAs were transinfected simultaneously. A blank plasmid harboring no siRNA was transfected as a blank control (sample 4). Normal fibroblasts were simultaneously detected in the normal group (baseline, sample 0 ). All transfected samples were validated by real-time PCR by comparing the relative expression to normal samples. All experiments were repeated three times.

\section{Systematic sequencing of IncRNAs, mRNAs and IncRNAs in five samples of keloid fibroblasts}

The sequences of IncRNAs, mRNAs and IncRNAs were measured in five samples of keloid fibroblasts. Briefly, total RNA or miRNA of respective groups of cells was abstracted (Invitrogen Life Technologies), amplified and transcribed into fluorescent cRNA (Arraystar, Rockville, MD). The labeled cRNAs were hybridized and deeply sequenced.

Differentially expressed (DE) mRNAs, IncRNAs and miRNAs in keloid fibroblasts either by HOXA11-AS knockdown or by nonspecific interference

DE profiles of mRNAs, IncRNAs or miRNAs were analyzed between HOXA11-AS-knockdown and normal keloid fibroblasts with threshold fold change $>1.5$ and P-value $\leq 0.05$. Keloid fibroblasts with HOXA11-AS knockdown (sample 1 and sample 2) or nonspecific interference were compared with normal keloid fibroblasts (sample 0) to analyze DE profiles. For DE profiles of mRNA or IncRNA in keloid fibroblasts with HOXA11-AS knockdown, an overlapping mRNA or IncRNA profile between sample 1 and sample 2 was intersected. For DE profiles of mRNA or IncRNA in keloid fibroblasts with nonspecific interference, a union profile between sample 3 and sample 4 was united. Outcome mRNAs or IncRNAs specifically targeting HOXA11-AS intersected DE mRNAs or IncRNAs, removing united mRNAs and IncRNAs.

\section{Bioinformatic analysis by construction of a IncRNA-mRNA-protein (LMP) interaction network to screen dominantly functioning proteins in keloid fibroblasts with HOXA11-AS knockdown}

Using bioinformatic prediction, two IncRNA-mRNA networks (LMNs) were constructed in keloid fibroblasts with HOXA11-AS knockdown or in keloid fibroblasts with nonspecific interference based on a human IncRNA-mRNA interrelated database (www.starbase.com) using a human protein-protein interaction (PPI) network as a "mediate" network. The rationale was that the dominant DE IncRNAs and DE mRNAs were both closely linked to PPI networks. Therefore, a proximity-based first neighbor network (FNN) was predicted in keloid fibroblasts with HOXA11-AS knockdown or in keloid fibroblasts with nonspecific interference. By this linkage, IncRNA-mRNA-protein (LMP) interaction networks were predicted in keloid fibroblasts with HOXA11-AS knockdown or in keloid fibroblasts with nonspecific interference. Within two LMP networks, proteins were ranked by the random-walk PageRank (PR) algorithm, which has been commonly used in calculating the importance of webpages being visited based on both count and quality, leading to PR-ranked proteins. Proteins were considered dominantly functioning proteins when their PR values were over average. Finally, the dominantly functional genes from the two networks were 
intersected in removing overlapping mRNAs that were attributive to nonspecific interference in keloid fibroblasts.

\section{Validation of screened genes by quantitative real-time polymerase chain reaction (PCR) and western blotting}

Total RNA was extracted from normal keloid fibroblasts and from keloid fibroblasts with HOXA11-AS knockdown or with nonspecific interference using an RNA extraction kit according to the manufacturer's protocol (Arraystar, Shanghai). Total cDNA was reverse transcribed from each sample using SuperScript III Reverse Transcriptase (Invitrogen). A standard curve was depicted by a diluted cDNA template gradient for each screened mRNA for qRT-PCR (Arraystar, Shanghai Kangcheng). The respective cRNA sample was used as a template for amplification. The sequences for primers of screened genes are shown in Supplementary Table S1. The qualitive concentration of each target gene was the relative percentage of gene concentration/GAPDH concentration. All real-time PCR have been conducted by three times.

For the validated mRNA(s), western blotting was further conducted in normal keloid fibroblasts and in keloid fibroblasts with HOXA11-AS knockdown. Western blotting was conducted according to routine protocols. SDS-PAGE (10\% gel) was used to separate the targeted proteins. Monoclonal antibodies, including anti-VTP (Abcam, ab113065, USA, diluted 1:300), anti-SNED1 (EartHOXA11, E021040, USA, diluted 1:4000), and anti-NIPAL3 (Abcam, ab32419, diluted 1:1000), were used for blotting capture.

\section{Biomathematics analysis by constructing a IncRNA-mRNA-miRNA competing endogenous RNA (ceRNA) network using IncRNA-mRNA interrelationships combining mRNA-miRNA network interrelationships}

For the screened mRNAs by real-time PCR and/or western blotting in keloid fibroblasts with HOXA11-AS knockdown, both IncRNA-mRNA interrelationships and mRNA-miRNA interrelationships were constructed. An expression-based competing endogenous interrelationship was downregulated IncRNA (knockdown of HOXA11-AS)-upregulated miRNA(s) (DE miRNAs)-downregulated mRNA(s) (all validated mRNAs). The DE miRNAs were identified between normal keloid fibroblasts (sample 0 ) and keloid fibroblasts with HOXA11-AS knockdown (sample 1 and sample 2). Among them, a sequence-based prediction of miRNAs as competitive sponges against the sequence of HOXA11-AS was further performed using the TargetScan 5.0 online tool. The outcome miRNAs undergoing expression and sequence dual validation were further identified by removing overlapping miRNAs that were also shown in the nonspecific group. DE miRNAs in the nonspecific group were obtained by union DE miRNAs between normal keloid fibroblasts (sample 0) and keloid fibroblasts with nonspecific interference (sample 4) and DE miRNAs between normal fibroblasts at keloid fibroblasts with blank plasmid interference (sample 5).

\section{Statistical Analysis}

All experiments were repeated three times. All data are displayed as the mean \pm standard deviation (S.D.). $P<0.05$ was used to show statistical significance. 


\section{Results}

Primarily cultured keloid fibroblasts in vitro from keloid explants in vivo

Keloid-derived fibroblasts were shown to be successfully grown in a few mini-keloid explants (4/22).

These four samples generally originated from relative edges of the keloid, indicating that central fibroblasts had been losing proliferative capability(14). Moreover, primary culturing presented a considerably slow growth curve. The slow proliferation of primarily cultured keloid fibroblasts reached its peak velocity after 10 days and then plateaued for a long time. Such a proliferation curve could reflect the confined hyperproliferation of keloids in vivo $(14,16)$.

Using optical microscopy, typical keloid fibroblasts were clearly visualized after 10 days of culture, with low-to-moderate proliferation (Fig. 1A). Notably, high nutritional culture medium (DMEM 15\% fetal bovine) was required to consistently sustain keloid fibroblasts with low proliferation. To display the extent of proliferation of these cultured keloid fibroblasts, a proliferative marker of Ki-67 antibody (green) was stained with nuclei stained with DAPI (blue). Using confocal microscopy, sparse Ki-17 positivity was shown in cultured keloid-derived fibroblasts (Fig. 1B). Globally, the growth of keloid fibroblasts is slow and sustainable.

\section{Validation of the siRNA interference of HOXA11-AS in keloid fibroblasts}

We validated the effect of interference of HOXA11-AS using real-time PCR between the two groups. It was significantly reduced in keloid fibroblasts with HOXA11-AS knockdown (Supplemental Fig. 1S), indicating that siRNA interference was successful.

\section{HOXA11-AS markedly altered the expression of mRNAs in keloid fibroblasts}

Heatmap expression (Fig. 2A and 2B) and DE profiling of keloid fibroblasts with HOXA11-AS knockdown (Fig. 2C) or with nonspecific interference (Fig. 2D) is shown. The counts of DE mRNAs in two keloid fibroblasts with HOXA11-AS knockdown (sample 1 and sample 2) were similar to those in normal keloid fibroblasts (sample 0). Intersection of the two sets produced a total of 1,396 DE mRNAs in keloid fibroblasts with HOXA11-AS knockdown. Figure 2B indicates that DE mRNAs in keloid fibroblasts with nonspecific interference were much higher than those with blank plasmid interference, indicating that nonspecific sequences could substantially interfere with gene profiling. The union of two sets produced 1,626 DE genes in keloid fibroblasts due to nonspecific interference.

\section{Hoxa11-as Mildly Altered Lncrna Expression In Keloid Fibroblasts}

Figure 3 included the heatmap express (Fig. 3A and 3B) and DE IncRNAs in keloid fibroblasts with HOXA11-AS knockdown (Fig. 3C) or with nonspecific interference (Fig. 3D). Only 39 DE IncRNAs were shown between keloid fibroblasts with HOXA11-AS knockdown (sample 1 and sample 2) and normal 
keloid fibroblasts (sample 0) (Fig. 3C). These DE IncRNAs were attributed to cross-talk between IncRNAs via an overlapping miRNA fine-tuning network. A total of 99 DE IncRNAs were shown in keloid fibroblasts with nonspecific interference combined with blank plasmid interference (Fig. 3D). All DE profiles are shown in Supplementary document $1 \mathrm{~S}$.

\section{Fourteen dominantly functioning genes in keloid fibroblasts with HOXA11-AS knockdown based on human IncRNA-mRNA-protein (LMP) interaction network prediction with PageRank scoring}

To screen potentially functional mRNAs regulated by HOXA11-AS in keloid fibroblasts, a stepwise bioinformatics analysis was performed. First, a IncRNA-mRNA network (LMN) was constructed using DE profiling in keloid fibroblasts with HOXA11-AS knockdown. The LMN network contained 39 IncRNA nodes (red) and 63 mRNA nodes (orange) (Fig. 4A upper). Second, using the aid of a human protein-proteininteraction network, proximity-based "neighbor" proteins were selected as dominant (green band). Third, the green band was integrated into the constructed LMN network to predict a IncRNA-mRNA-protein (LMP) network (Fig. 4B upper). The LMP network contained 929 nodes and 6,572 edges, including the constructed LMN (39 IncRNA, red node; 63 mRNA, orange node) and first-step dominant genes (green band). Fourth, using the advanced PageRank (PR) random walk algorithm, all screened functional mRNAs were scored. A total of 23 mRNAs ranked above the average PR score were screened as dominantly functioning genes in keloid fibroblasts by HOXA11-AS knockdown (Fig. 4C upper). Next, the biological enrichments removed 7 unenriched mRNAs from 23 screened mRNAs in keloid fibroblasts with HOXA11-AS knockdown, leading to 16 dominantly functioning proteins in keloid fibroblasts with HOXA11AS knockdown (Fig. 4D upper). Thus, HOXA11-AS might regulate 16 genes that function in keloid fibroblast proliferation.

To remove nonspecific interference, using the same protocol, an LMN containing 28 IncRNAs and 30 mRNAs was predicted using DE profiling in keloid fibroblasts by nonspecific interference (Fig. 4A, lower). An integrated LMP network was then constructed (Fig. 4B lower). A total of 14 mRNAs characterized by PageRank above the average score were screened in keloid fibroblasts by nonspecific interference (Fig. 4C lower). As pathway enrichment was meaningless for nonspecific interference, all 14 screened mRNAs remained the next step (Fig. 4D lower). After two mRNAs that emerged in both keloid fibroblasts by HOXA11-AS knockdown and in keloid fibroblasts with nonspecific interference, they were removed as potential "noise". Consequently, a total of 14 mRNAs were thought to function as targets in the HOX11AAS-involved molecular network during keloid formation (Fig. 4E).

\section{Pathway enrichment of 14 putatively functioning mRNAs in keloid fibroblasts by HOX11A-AS knockdown}

Using Gene Ontology (G0) and KEGG online analysis, 14 putative functional genes (VTN, BAG4, PSMD7, SNED1, SUPT6H, POMZP3, FOXM1, TMEM204, GNG5, NIPAL3, TFPT, CYB5RL, CHD3 and RPS27A) were significantly enriched in three pathways, i.e., UCH proteinases, NABA ECM GLYCOPROTEINS and cellular response to growth factor stimulus (Fig. 5A). This result meant that H0X11A-AS upstream of the regulatory pathway strain could extensively participate in the function, albeit subtle and slight. 
Considering the long noncoding trait, these genes were supposed to be indirectly impacted within an enormous network, some of which were similar to Domino downstream.

\section{Validation of candidate mRNAs in keloid fibroblasts by HOX11A-AS knockdown using qPCR and western blotting}

Using quantitative PCR, three genes (SED1, NIPAL3 and VTN) were shown to be significantly changed between keloid fibroblasts with HOXA11-AS knockdown and normal keloid fibroblasts. Therefore, these genes were supposed to be the dominant targets downstream of the HOXA11-AS1 regulatory pathway (Fig. 5B). Original datasets and curves of these fourteen qPCR assays are available on request.

However, western blotting detection showed negative expression of SED1, NIPAL3 and VTN either in normal keloid fibroblasts or in keloid fibroblasts with HOXA11-AS knockdown. These negative results were not shown. As all three genes belonged to endogenously expressed superfamilies with very low expression, trace expression could not be detected using western blotting because it harbors much lower sensitivity but higher specificity than PCR.

\section{Screening potential molecular sponging miRNAs for HOXA11-AS to regulate three validated genes by competing endogenous RNA (ceRNA) network in keloid fibroblasts}

For three validated three genes with very low expression in keloid fibroblasts, a question arose: how are these genes regulated by HOXA11-AS? One of the most common regulatory mechanisms is the expression of IncRNAs combined with miRNA(s) to attenuate the inhibitory sponging effects of miRNAs on target genes. Therefore, we attempted to conduct another bioinformatic analysis to screen potential sponging miRNAs in keloid fibroblasts (Fig. 6).

First, we predicted 126 sequence-matched miRNAs that might interact with NIPAL3 (104 miRNAs), SNED1 (13 miRNAs) and VTN (9 miRNAs), referring to the human mRNA-miRNA competing endogenous RNA database among the DE miRNA profiles $(2,17)$. Simultaneously, we conducted expression-based molecular interrelationships to identify the HOXA11-AS molecular pair. According to Down (HOXA11-AS knockdown)-Up (DE miRNAs)-Down (validated genes) in keloid fibroblast, upregulated VTN was removed, remaining downregulated NIPAL3 and SNED1. Sequence-based validation and expression-based dual validation addressed 10 upregulated candidate miRNAs with sequences competing with NIPAL3 among the DE miRNA profiles in keloid fibroblasts.

To further remove nonspecific interference, DE miRNA profiling in keloid fibroblasts with nonspecific interference was analyzed, obtaining 495 upregulated DE miRNAs (Supplementary List 1). Among them, 7 miRNAs that also emerged in 10 candidate miRNAs were removed as nonspecific interference.

Consequently, three upregulated miRNAs, including hsa-miR-19a-3p, hsa-miR-141-3p, and hsa-miR-140$5 p$, were predicted as molecular sponges to specifically downregulate the expression of NIPAL3 by the downregulation of HOXA11-AS in keloid fibroblasts. 


\section{Discussion}

Our previous study revealed a significantly increased expression of HOXA11-AS in keloids by array-based IncRNA comparison (10). In the present study, we further attempted to reveal the potential regulation of potential miRNA targets involved in HOXA11-AS during keloid formation in vitro.

When we attempted to culture keloid fibroblasts primarily from in vivo keloid explants, we observed slow in vitro proliferation post 10 days and then plateaued without a hyperproliferation peak during the 50 days scenario. Moreover, a high nutritional microenvironment was required to sustain slow proliferation. Therefore, we supposed that the excessive proliferation of keloids ceased due to lower nutritional status. Ki-67 staining validated the weak capability of proliferation even with high nutritional supply. Taken together, we hypothesized that the in vitro primarily cultured keloid fibroblasts could reflect the subtle regulation between differentiation and proliferation in vivo.

We next investigated the function of the IncRNA HOXA11-AS in the keloid fibroblasts using a routine lossof-function design. We simultaneously transfected three siRNAs to "knockdown" the expression of HOXA11-AS in keloid fibroblasts; however, we could only significantly downregulate the expression of HOXA11-AS in keloid fibroblasts. To reduce the "noise" from nonspecific interference, we constructed plasmids harboring nonspecific sequences for transfection and plasmids harboring blank plasmids for transfection. To enhance the specificity of the bioinformatics-based outcome, we removed the union profiles of both the nonspecific group and the blank group.

We then conducted a stepwise bioinformatic analysis. First, we obtained three DE profiles (IncRNA, mRNA and miRNA) by knocking down HOXA11-AS or by nonspecific interference. We removed overlapping DE molecules between the two groups to remove "noise". Then, we constructed a IncRNA-mRNA predictive network to screen dominant mRNAs downstream of the HOXA11-AS pathway. Our rationale was that spatiotemporal proximity meant functional dominance. By this step, we screened 14 dominant mRNAs among DE mRNAs as candidate functional genes in the HOXA11-AS-knockdown keloid fibroblasts. Intriguingly, these genes were largely endogenous genes enriched in three pathways: one pathway on cellular response to growth factor stimulus and two pathways on transcriptional regulation of endogenous genes. Using cellular validation, merely three genes (NIPAL3, SNED1, VNT) were found to be significantly changed among the 14 candidate genes in keloid fibroblasts via real-time PCR. No protein of these genes could be detected by western blotting even in normal keloid fibroblasts without any molecule knockdown, largely attributive to the trace expression of these endogenous genes.

Supposing NIPAL3, SNED1, and VNT are targets downstream of HOXA11-AS, we attempted to identify miRNA "mediators" by prediction of the ceRNA network. It is well accepted that endogenous competing miRNAs imperatively regulate cellular processes through their 3'-UTR binding to respective target mRNAs. We predicted sequence-based competing matching network using a public database (18) (19). Also, we predicted expression-based matching network on DE profiles. The present expression-based network was downregulated (HOXA11-AS knockdown)-upregulated (among upregulated DE miRNAs)-downregulated (PCR-validated NIPAL3 and SNED1) in keloid fibroblasts. The dual validation had identified ten 
upregulated sponging miRNAs against one gene of NIPAL3. After removing seven miRNAs that were also differentially expressed in keloid fibroblasts with nonspecific interference, we constructed a final ceRNA network characterized by downregulated HOXA11-AS, three upregulated miRNAs (hsa-miR-19a-3p, hsamiR-141-3p and hsa-miR-140-5p), and reduced NIPAL3 in keloid fibroblasts.

In previous studies, the aberrative expression of HOXA11-AS was shown to correlate with a bulk of functions, including proliferation, migration, invasion and epithelial mesenchymal transition, via dozens of different miRNAs, presenting hundreds of regulatory pathways in various tumors (reviewed by 11). Dozens of miRNAs have been documented in various cancers, indicating that HOXA11-AS participates in a widely involved molecular network. Here, we used keloid-derived fibroblasts in vitro to explore the HOXA11-AS-involved molecular network. NIPAL3 had been previously annotated to regulate magnesium ion transmembrane transporters, which is fundamental to cellular biology. Three miRNAs (hsa-miR-19a$3 p$, hsa-miR-141-3p and hsa-miR-140-5p) were previously predicted as sponging meditators in various cancers. Among them, miR-19a-3p was shown to not only promote epithelial mesenchymal transition (EMT) and metastasis in some cancers (20-24) but also inhibit EMT in vitro(25) and in other types of cancers(26-29). Both miR-140-5p and miRNA-141-3p have been shown to inhibit EMT in numerous tumors $(26,30-37)$. As EMT plays an essential role in keloid formation(38-40), such complicated miRNA cooperation of hsa-miR-19a-3p, hsa-miR-141-3p and hsa-miR-140-5p could aid HOXA11-AS in subtle balancing EMT during keloid tumorigenesis $(12,13)$. Additionally, these "paradoxical" miRNAs highly consisted of the "paradoxical" regulation of HOXA1-AS in various cancers.

Three remarkable strengths of the research were as follows: first, three DE profiles (IncRNAs, mRNAs and miRNAs) were systematically sequenced with synchronicity; second, siRNA knockdown of HOXA11-AS was performed with complete nonspecific interference control to reduce "noise" during high-throughput data analysis; third, advanced bioinformatics analysis was performed to construct a IncRNA-miRNAmRNA network from interior interrelationships, with high respect to the integrative complexity of molecular network regulation; and fourth, the miRNA-mRNA pairs were dually validated by expression and sequence, avoiding subjective bias.

Our studies have several limitations. First, the validation sample size was small. Second, western blotting could not detect protein-level alterations even in normal keloid fibroblasts without any knockdown, indicating very low expression of the target gene. Third, case-specific bioinformatic analysis lacked a standard protocol for high-quality control.

To the best of our knowledge, our findings were the first to prove that multiple miRNA networks are involved in ceRNA with potentially "opposite" buffing regulation. More studies are required to investigate the subtle "balance" of these sponging miRNAs in the HOXA11-AS-dependent network to reveal the mechanism of keloid fibroblasts.

\section{Conclusions}


HOXA11-AS was predicted to subtly regulate 14 genes in keloid fibroblasts. Among them, three mRNAs (NIPAL3, SNED1 and VTN) were validated by quantitative PCR. Three miRNAs (hsa-miR-19a-3p, hsa-miR141-3p and hsa-miR-140-5p) were predicted as sponging molecules to downregulate NIPAL3 in HOXA11AS regulatory pathway in keloid fibroblasts.

\section{Abbreviations}

DE

Differentially Expressed

EMT

Epithelial-Mesenchymal Transition

IncRNA

long noncoding RNA

miRNA

microRNA

mRNA

message RNA

PCR

Polymerase Chain Reaction

PR

PageRank

\section{Declarations}

Ethics approval and consent to participateIThe study was approved by the Medical Ethics committee of China-Japan Union Friendship Hospital of Jilin University, Changchun, China. All patients were informed with written consent. The study was conducted in accordance with the Declaration of Helsinki Principles.

Consent for publication凹Not applicable.

Availability of data and materials $\square$ All data generated or analysed during this study are included in this published article [Supplementary files].

Competing interests[The authors declare that they have no competing interests

Funding $\square T h e$ work was funded by The Group Research Project in Jilin University (No. 2018YX019)

\section{Authors' contributions [}

Dr. Qiang Wang: Design the study, Interpretation of data, Draft writing

Dr. Wei Wang: Design the study, Interpretation of data, Draft writing

Dr. Xiaojie Sun: Design the study, Interpretation of data, Draft writing and revising 


\section{Acknowledgements $\square$}

We thank for Dr. Yuhong Wang's insightful aid on the manuscript writing.

\section{References}

1. Macarak EJ, Wermuth PJ, Rosenbloom J, Uitto J. Keloid disorder: Fibroblast differentiation and gene expression profile in fibrotic skin diseases. Exp Dermatol. 2021;30:132-45.

2. Alizadeh Z, et al. Effect of intramural myomectomy on endometrial HOXA10 and HOXA11 mRNA expression at the time of implantation window. Iran J Reprod Med. 2013;11:983-8.

3. Guan G, et al. Upregulation of Neural Cell Adhesion Molecule 1 (NCAM1) by hsa-miR-141-3p Suppresses Ameloblastoma Cell Migration. Medical science monitor: international medical journal of experimental clinical research. 2020;26:e923491.

4. Langellotto $F$, et al. Expression of meis and hoxa11 in dipnoan and teleost fins provides new insights into the evolution of vertebrate appendages. Evodevo. 2018;9:11.

5. Bai F, et al. Simvastatin induces breast cancer cell death through oxidative stress up-regulating miR140-5p. Aging. 2019;11:3198-219.

6. Anfossi S, et al. High serum miR-19a levels are associated with inflammatory breast cancer and are predictive of favorable clinical outcome in patients with metastatic HER2 + inflammatory breast cancer. PloS one. 2014;9:e83113.

7. Bai R, et al. The NF-kB-modulated miR-19a-3p enhances malignancy of human ovarian cancer cells through inhibition of IGFBP-3 expression. Molecular carcinogenesis. 2019;58:2254-65.

8. Chen $\mathrm{D}$, et al. Targeting the radiation-induced TR4 nuclear receptor-mediated QKI/circZEB1/miR-1413p/ZEB1 signaling increases prostate cancer radiosensitivity. Cancer letters. 2020;495:100-11.

9. Su X, Ma Y, Wang Q, Gao Y. LncRNA HOXA11-AS Aggravates Keloid Progression by the Regulation of HOXA11-AS-miR-205-5p-FOXM1 Pathway. J Surg Res. 2021;259:284-95.

10. Chen $D$, et al. The IncRNA HOXA11-AS functions as a competing endogenous RNA to regulate PADI2 expression by sponging miR-125a-5p in liver metastasis of colorectal cancer. Oncotarget. 2017;8:70642-52.

11. Lu CW, et al. HOXA11 antisense long noncoding RNA (HOXA11-AS): A promising IncRNA in human cancers. Cancer medicine. 2018;7:3792-9.

12. Yim GW, et al. Long Non-coding RNA HOXA11 Antisense Promotes Cell Proliferation and Invasion and Predicts Patient Prognosis in Serous Ovarian Cancer. Cancer research treatment. 2017;49:65668.

13. Richards EJ,, et al. A functional variant in HOXA11-AS, a novel long non-coding RNA, inhibits the oncogenic phenotype of epithelial ovarian cancer. Oncotarget. 2015;6:34745-57.

14. Luo S, Benathan M, Raffoul W, Panizzon RG, Egloff DV. Abnormal balance between proliferation and apoptotic cell death in fibroblasts derived from keloid lesions. Plast Reconstr Surg. 2001;107:87-96. 
15. Tucci-Viegas VM, Hochman B, Franca JP, Ferreira LM. Keloid explant culture: a model for keloid fibroblasts isolation and cultivation based on the biological differences of its specific regions. Int Wound J. 2010;7:339-48.

16. Ashcroft KJ, Syed F, Bayat A. Site-specific keloid fibroblasts alter the behaviour of normal skin and normal scar fibroblasts through paracrine signalling. PloS one. 2013;8:e75600.

17. Chen $M$, Lin $M$, Wang X. Overexpression of miR-19a inhibits colorectal cancer angiogenesis by suppressing KRAS expression. Oncol Rep. 2018;39:619-26.

18. Cui Y, Yi L, Zhao JZ, Jiang YG. Long Noncoding RNA HOXA11-AS Functions as miRNA Sponge to Promote the Glioma Tumorigenesis Through Targeting miR-140-5p. DNA cell biology. 2017;36:8228.

19. Qiu W, Kassem M. miR-141-3p inhibits human stromal (mesenchymal) stem cell proliferation and differentiation. Biochim Biophys Acta. 2014;1843:2114-21.

20. Li Z, Li Y, Wang Y. miR-19a promotes invasion and epithelial to mesenchymal transition of bladder cancer cells by targeting RhoB. Journal of BUON: official journal of the Balkan Union of Oncology. 2019;24:797-804.

21. Li J, et al. MicroRNA-19 triggers epithelial-mesenchymal transition of lung cancer cells accompanied by growth inhibition. Lab Invest. 2015;95:1056-70.

22. Huang $L$, et al. Hsa-miR-19a is associated with lymph metastasis and mediates the TNF-a induced epithelial-to-mesenchymal transition in colorectal cancer. Scientific reports. 2015;5:13350.

23. Lu WD, Zuo Y, Xu Z, Zhang M. MiR-19a promotes epithelial-mesenchymal transition through PI3K/AKT pathway in gastric cancer. World journal of gastroenterology. 2015;21:4564-73.

24. Li D, Liu K, Li Z, Wang J, Wang X. miR-19a and miR-424 target TGFBR3 to promote epithelial-tomesenchymal transition and migration of tongue squamous cell carcinoma cells. Cell adhesion migration. 2018;12:236-46.

25. Ye Q, et al. MicroRNA-141 inhibits epithelial-mesenchymal transition, and ovarian cancer cell migration and invasion. Mol Med Rep. 2017;16:6743-9.

26. Zhang $Y$, et al. Down-regulation of IncRNA-ATB inhibits epithelial-mesenchymal transition of breast cancer cells by increasing miR-141-3p expression. Biochemistry cell biology = Biochimie et biologie cellulaire. 2019;97:193-200.

27. Tamagawa S, et al. Role of miR-200c/miR-141 in the regulation of epithelial-mesenchymal transition and migration in head and neck squamous cell carcinoma. Int J Mol Med. 2014;33:879-86.

28. Huang $S$, et al. Downregulation of miR-141-3p promotes bone metastasis via activating NF-KB signaling in prostate cancer. Journal of experimental clinical cancer research: CR. 2017;36:173.

29. Chen L, et al. Upregulation of microRNA-141 suppresses epithelial-mesenchymal transition and lymph node metastasis in laryngeal cancer through HOXC6-dependent TGF- $\beta$ signaling pathway. Cellular signalling. 2020;66:109444. 
30. Li C, et al. TGFß1- miR-140-5p axis mediated up-regulation of Flap Endonuclease 1 promotes epithelial-mesenchymal transition in hepatocellular carcinoma. Aging. 2019;11:5593-612.

31. Li C, et al. TGFbeta1- miR-140-5p axis mediated up-regulation of Flap Endonuclease 1 promotes epithelial-mesenchymal transition in hepatocellular carcinoma. Aging. 2019;11:5593-612.

32. Liu W, Liu P, Gao H, Wang X, Yan M. Long non-coding RNA PGM5-AS1 promotes epithelialmesenchymal transition, invasion and metastasis of osteosarcoma cells by impairing miR-140-5pmediated FBN1 inhibition. Molecular oncology. 2020;14:2660-77.

33. Tang $\mathrm{YH}_{\text {, }}$ et al. The long noncoding RNA AK002107 negatively modulates miR-140-5p and targets TGFBR1 to induce epithelial-mesenchymal transition in hepatocellular carcinoma. Molecular oncology. 2019;13:1296-310.

34. Lv J, et al. Long non-coding RNA Unigene56159 promotes epithelial-mesenchymal transition by acting as a ceRNA of miR-140-5p in hepatocellular carcinoma cells. Cancer letters. 2016;382:16675.

35. Fan L, et al. Long Noncoding RNA MALAT1 Contributes to Sorafenib Resistance by Targeting miR140-5p/Aurora-A Signaling in Hepatocellular Carcinoma. Mol Cancer Ther. 2020;19:1197-209.

36. Zhai H, Fesler A, Ba Y, Wu S, Ju J. Inhibition of colorectal cancer stem cell survival and invasive potential by hsa-miR-140-5p mediated suppression of Smad2 and autophagy. Oncotarget. 2015;6:19735-46.

37. Zheng $L$, et al. ELF3 promotes epithelial-mesenchymal transition by protecting ZEB1 from miR-1413p-mediated silencing in hepatocellular carcinoma. Cell death disease. 2018;9:387.

38. Yang $\mathrm{CE}_{\text {, }}$ et al. Epithelial-mesenchymal transition in keloid tissue. Arch Plast Surg. 2018;45:600-1.

39. Hahn JM, McFarland KL, Combs KA, Supp DM. Partial epithelial-mesenchymal transition in keloid scars: regulation of keloid keratinocyte gene expression by transforming growth factor-beta1. Burns Trauma. 2016;4:30.

40. Kuwahara $\mathrm{H}$, et al. Examination of Epithelial Mesenchymal Transition in Keloid Tissues and Possibility of Keloid Therapy Target. Plast Reconstr Surg Glob Open. 2016;4:e1138.

\section{Figures}


A
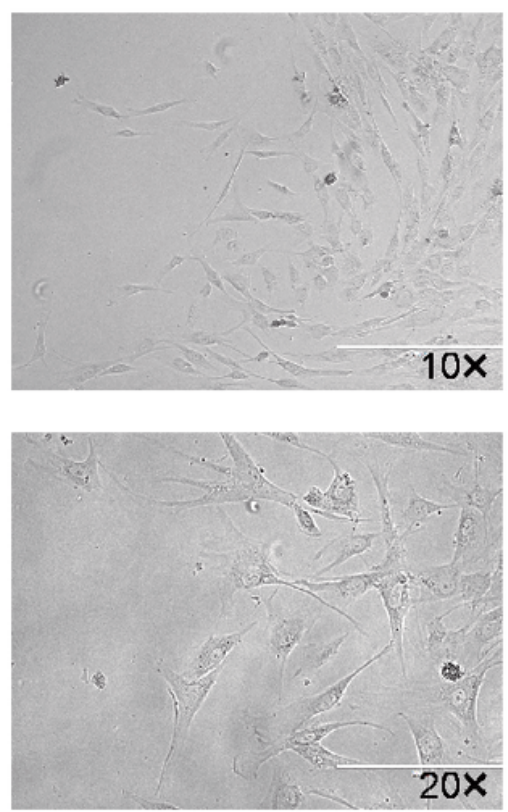

B
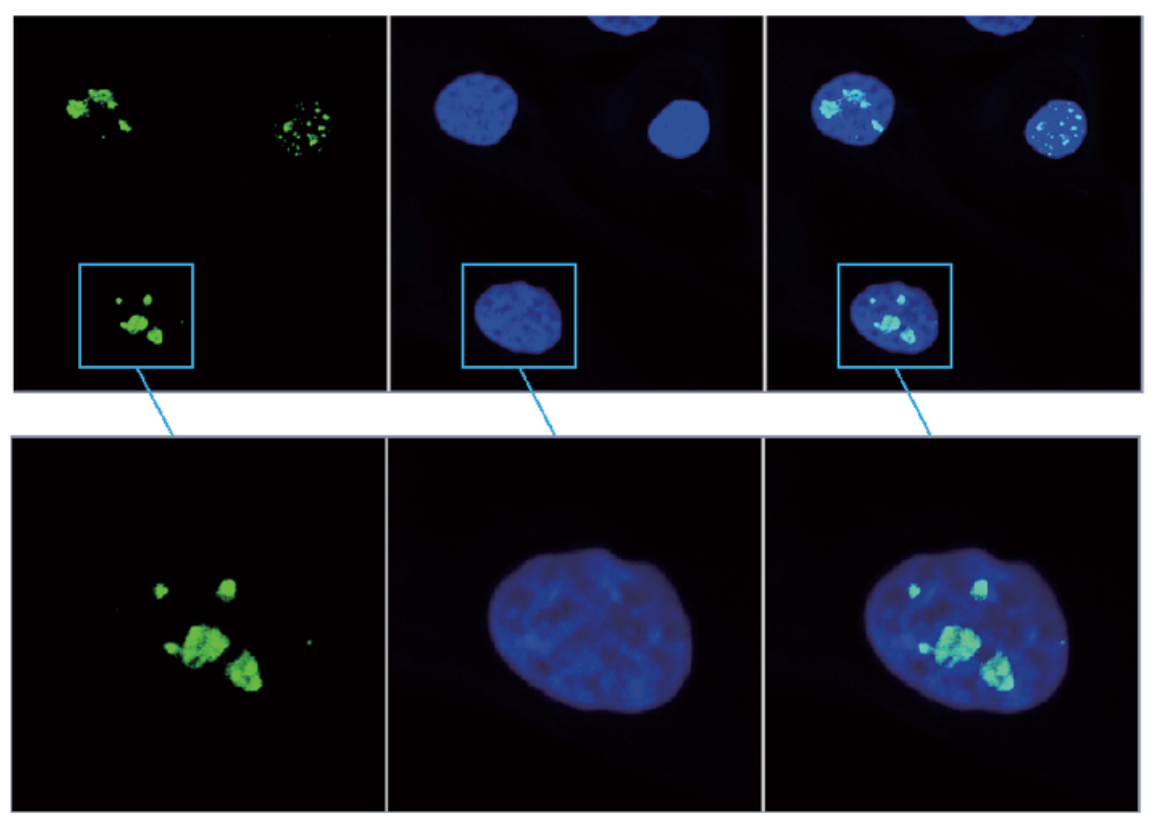

Ki67

DIPA

Merge

\section{Figure 1}

Primary culture of keloid fibroblasts A. Typical keloid fibroblasts were observed via optical microscopy post 15 days of primary culture from keloid explants. (Bar: $200 \mu \mathrm{m})$. B. Using Ki-67 as a marker of cell proliferation, it was shown that low-to-moderate Ki-67 (green) and DAPI-stained cell nuclei (blue) were coexpressed in keloid fibroblasts via confocal microscopy post 15 days of primary culture from keloid explants. 
A

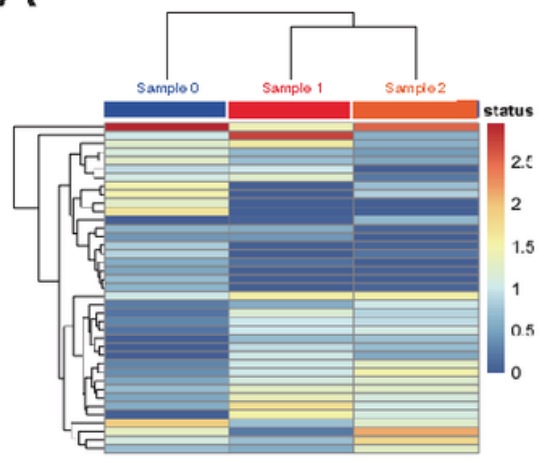

B

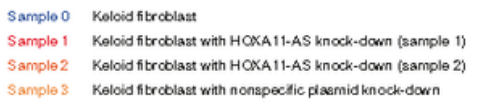
Sample 2 Keloid fitroblast with HOXA11-AS knock-down (sample 2) Keloid fitrcolast with blank plasmid knock-down

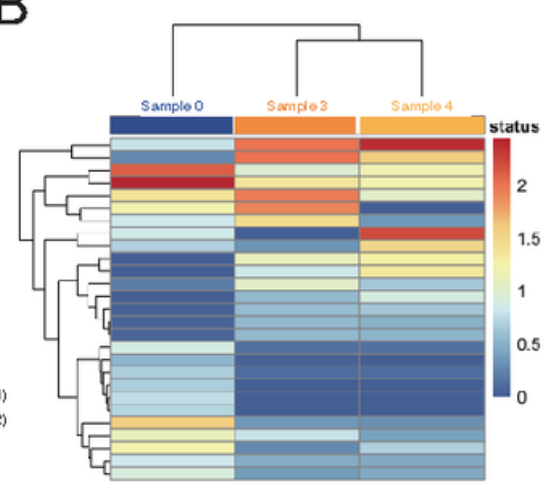

Bioinformatic analysis of Differentially Expressed (DE) IncRNAs
C
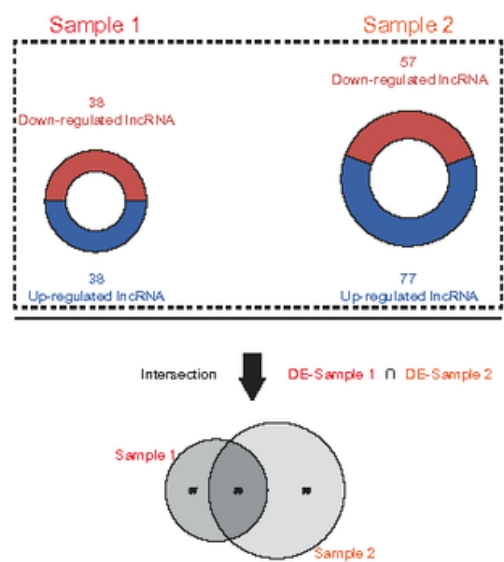

Differential expressed IncRNAs in keloid fibroblast [HOXA11-AS knockdown]

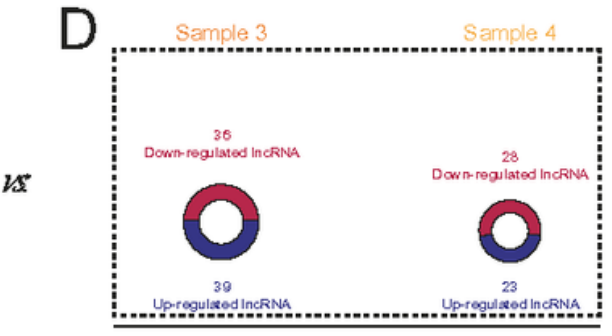

Union

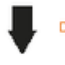

Sample

Differential expressed IncRNAs in keloid fibroblast [nonspecific interference]

\section{Figure 2}

Message RNA (mRNA) expression heatmap and differentially expressed profiles in keloid fibroblasts Message RNA (mRNA) expression heatmap of sample 0, sample 1 and sample 2 (Figure 2A) as well as sample0, sample 3 and sample 4 (Figure 2B) were shown. The differentially expressed (DE) mRNA profiles between sample 0 and sample 1 as well as between sample 0 and sample 2 were intersected to obtain DE profiles in keloid fibro blasts with HOXA11-AS knockdown (Figure 2C). The differentially expressed (DE) mRNA profiles between sample 0 and sample 1 as well as between sample 0 and sample 2 were united to obtain DE profiles in keloid fibroblasts with nonspecific interference (Figure 2D). 

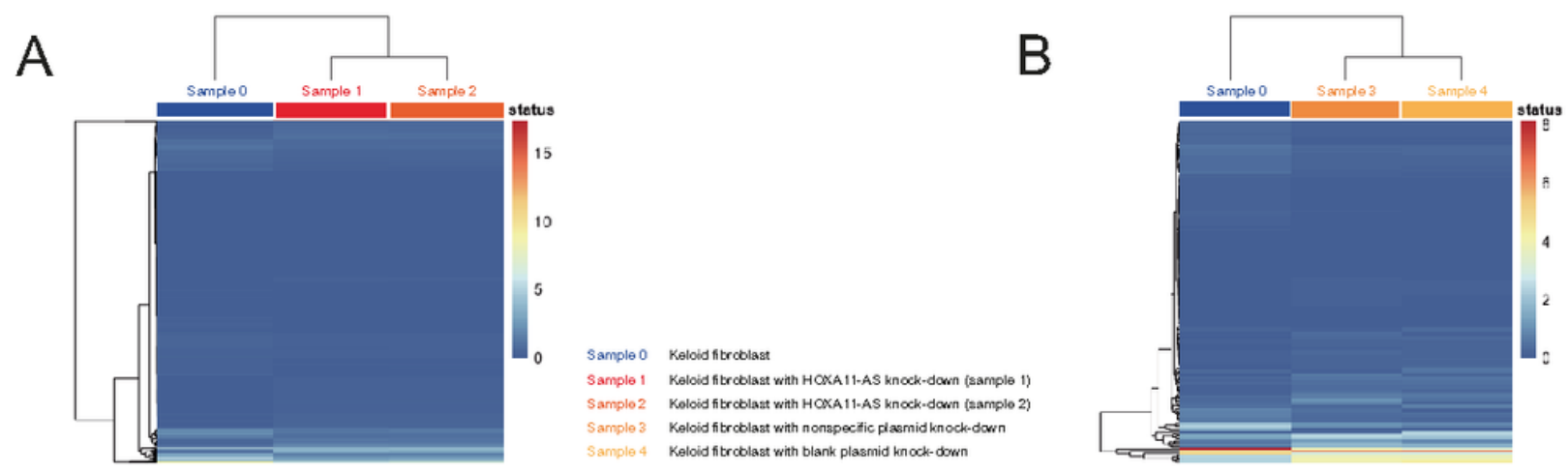

Bioinformatic analysis of Differentially Expressed (DE) mRNAs

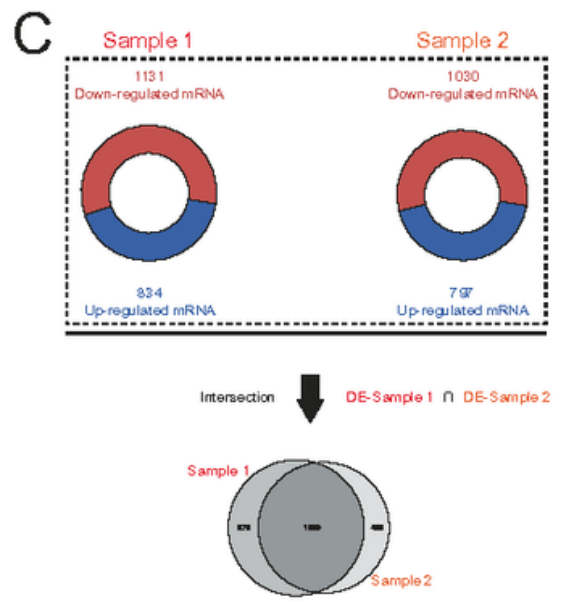

Differential expressed mRNAs in keloid fibroblast [HOXA11-AS knockdown]

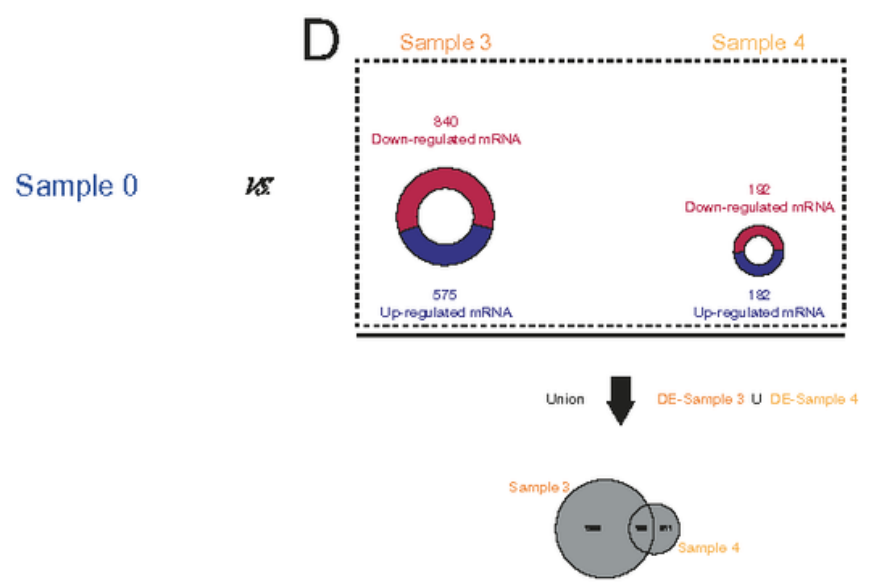

Differential expressed mRNAs in keloid fibroblast [nonspecific interference]

\section{Figure 3}

Long noncoding RNA (IncRNA) expression heatmap and differentially expressed profiles in keloid fibroblasts Long noncoding RNA (IncRNA) expression heatmap of sample 0 , sample 1 and sample 2 (Figure $3 \mathrm{~A}$ ) as well as sample0, sample 3 and sample 4 (Figure 3B) were shown. The differentially expressed (DE) mRNA profiles between sample 0 and sample 1 as well as between sample 0 and sample 2 were intersected to obtain DE profiles in keloid fibro blasts with HOXA11-AS knockdown (Figure 3C). The differentially expressed (DE) mRNA profiles between sample 0 and sample 1 as well as between sample 0 and sample 2 were united to obtain DE profiles in keloid fibroblasts with nonspecific interference (Figure 3D). 


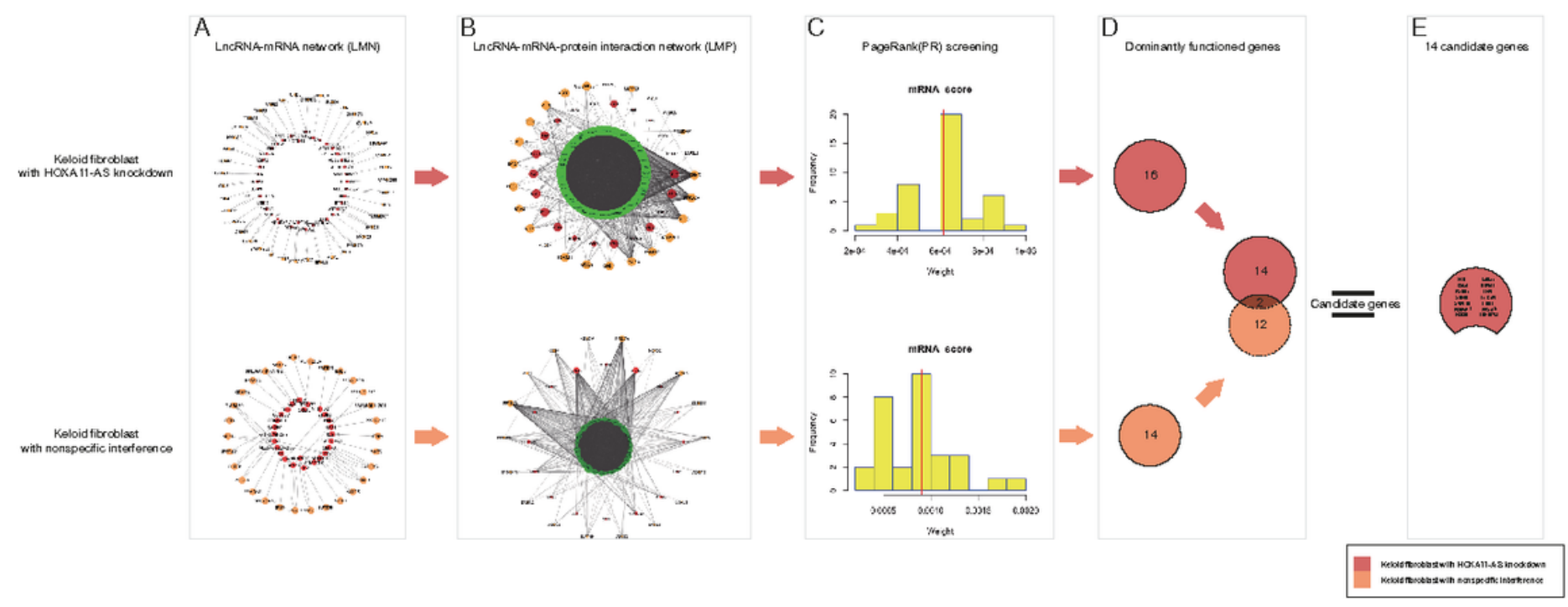

\section{Figure 4}

Predicting dominantly functioned mRNAs regulated by HOXA11-AS in keloid fibroblasts using coexpression network construction A IncRNA-mRNA network (LMN) was constructed using differentially expressed (DE) IncRNAs and DE mRNAs in keloid fibroblasts with HOXA11-AS knockdown (Figure 4A Upper). A total of $39 \mathrm{DE}$ IncRNAs (red nodes) corresponding to $63 \mathrm{DE}$ mRNAs (orange nodes) were predicted by knocking down HOXA11-AS in keloid fibroblasts. Using the same protocol, LMN in keloid fibroblasts with nonspecific interference was constructed (Figure 4A Lower). A total of 28 DE IncRNAs (red nodes) corresponding to $30 \mathrm{DE}$ mRNAs (orange nodes) were predicted in keloid fibroblasts by nonspecific interference. An integrated IncRNA-mRNA-protein (LMP) network was constructed based on the rationale that proximity predicts functional dominance. Therefore, the first-step genes (green band) linked to 39 IncRNAs (red nodes) and to 63 mRNAs (orange nodes) were identified using DE mRNA profile in keloid fibroblasts with HOXA11-AS knockdown (Figure 4A Upper) based on a human protein-proteininteraction (PPI) annotated network. Using the same protocol, the integrated LMP network was constructed in keloid fibroblasts with nonspecific interference (Figure 4B Lower). Dominantly functioned mRNAs were screened by the PageRank (PR) algorithm. A total of 23 mRNAs were predicted in keloid fibroblasts with HOXA11-AS knockdown (Figure 4C Upper). Interestingly, 14 mRNAs were also predicted in keloid fibroblasts with nonspecific interference (Figure 4C Lower). Using functional enrichment, 16 screened mRNAs from 23 mRNAs were enriched in the HOXA11-AS pathway in keloid fibroblasts (Figure 4D Upper). No functional enrichment was conducted for the nonspecific group (Figure 4D Lower). Among 16 screened mRNAs that were predicted to be involved in the HOXA11-AS pathway, 2 mRNAs that were also predicted to be involved in nonspecific interference were removed as "noise", leaving 14 candidate mRNAs regulated by HOXA11-AS in keloid fibroblasts (Figure 4E). 
A

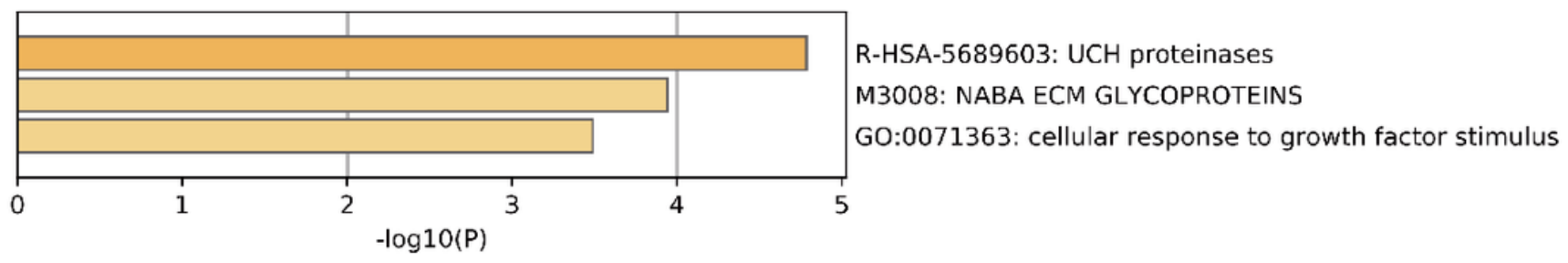

GO analysis of HOXA11-AS in keloid fibroblast

B
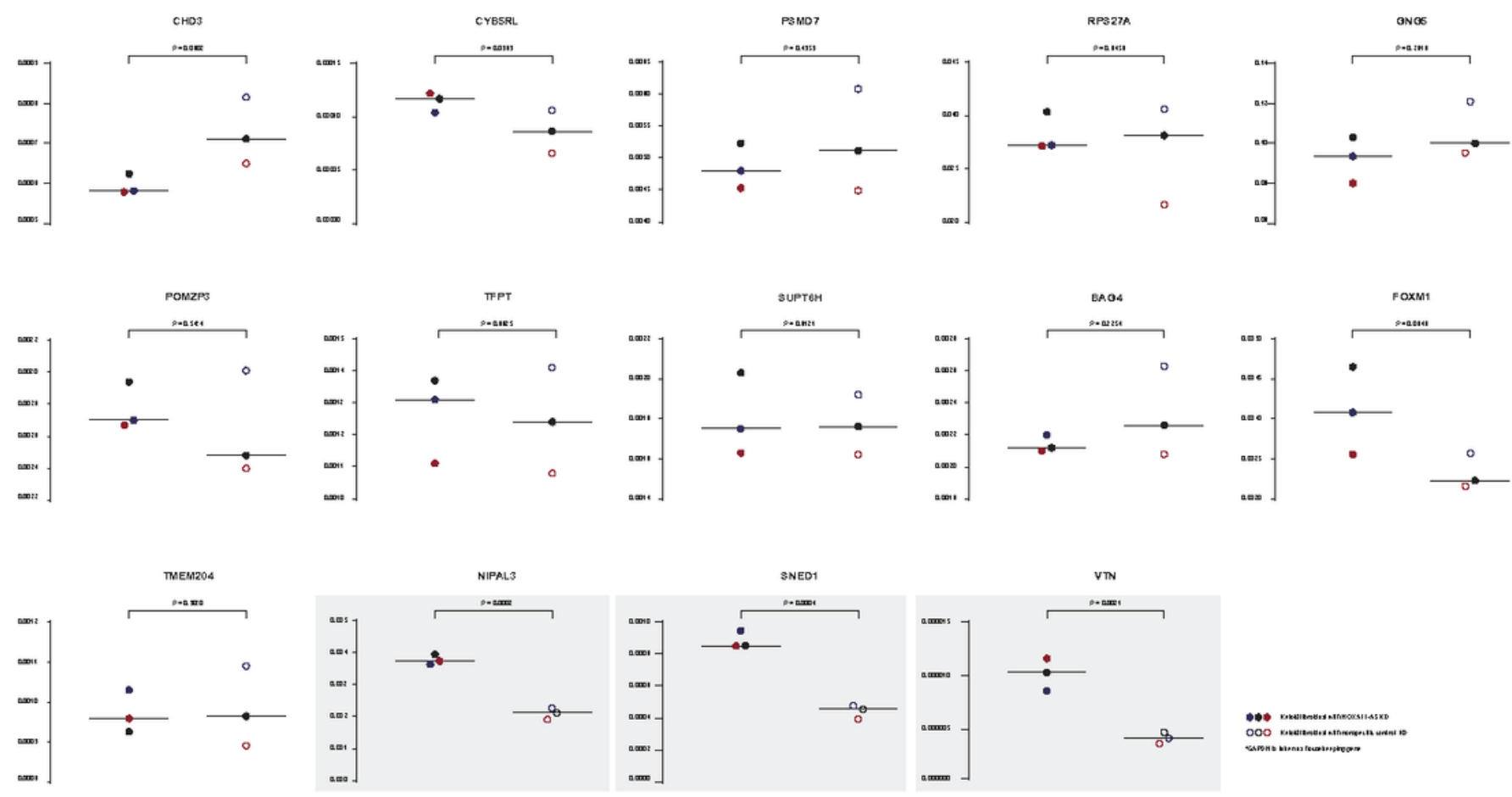

Relative expression of 14 mRNAs via real-time PCR in keloid fibroblast with or withoutOXA11-AS

\section{Figure 5}

Pathway enrichment of 14 mRNAs and PCR validation in keloid fibroblasts with HOXA11-AS knockdown Three pathways were enriched in 14 putative candidate mRNAs in keloid fibroblasts involved in the HOXA11-AS pathway using GO online analysis (Figure 5A). Three mRNAs, VTN, SNED1 and NIPAL3, were found to be significantly changed in keloid fibroblasts with HOXA11-AS knockdown compared with normal controls using qPCR validation (Figure 5B). 
A

Sequence-based ceRNA network

mRNA-miRNA

miRNA.IIORNA

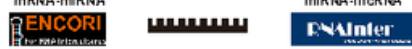

C

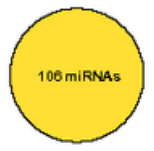

B

Expression-based ceRNA network

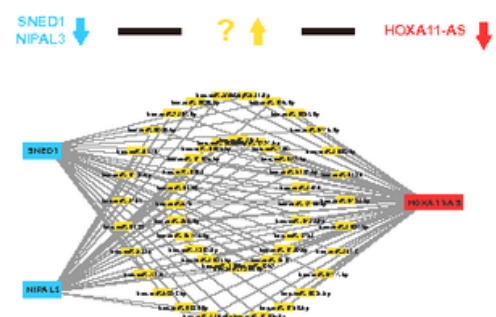

Differentially Expressed (DE) miRNAs in keloid fibroblast

with HOXA11AS knockdown

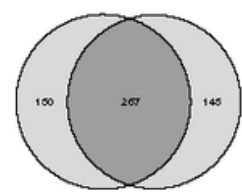

D

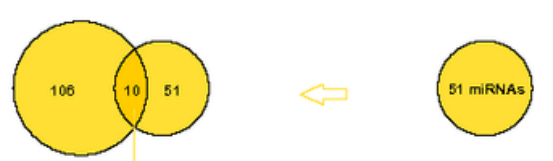

Differentially Expressed (DE) miRNAs

in keloid fibroblast
with nonspecific interference
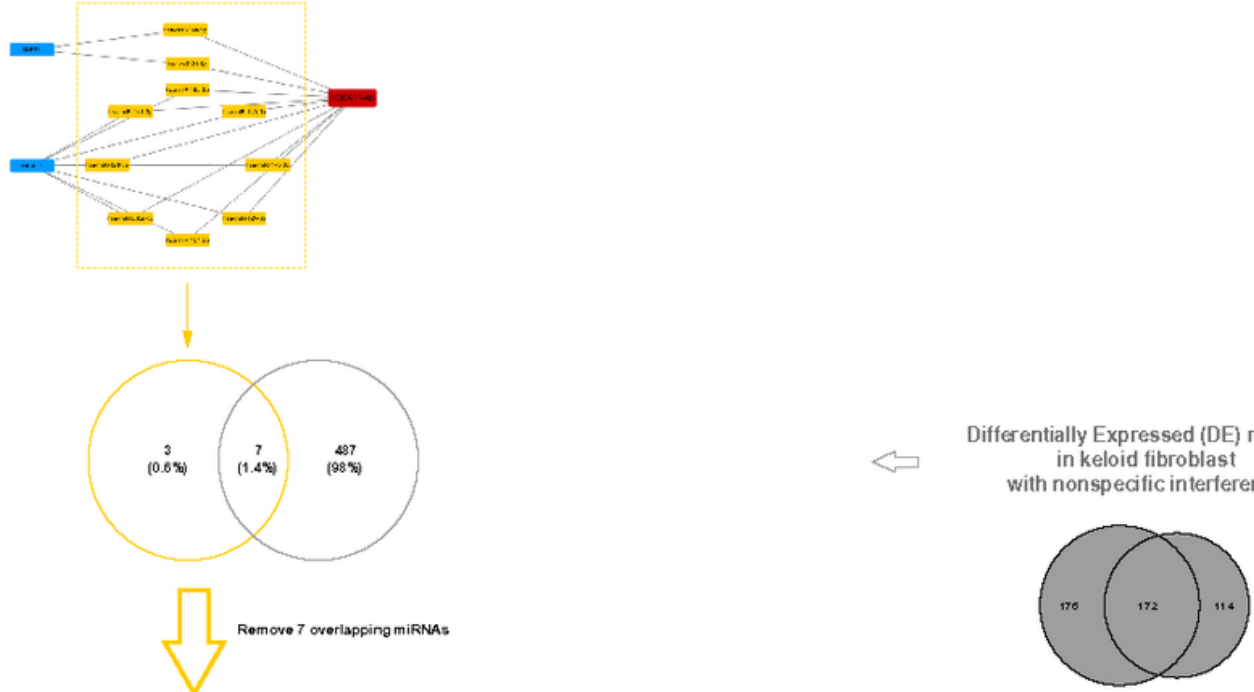

\section{Figure 6}

Construction of a competing endogenous RNA (ceRNA) network for HOXA11-AS in keloid fibroblasts Competing endogenous miRNAs were screened for sequence-based prediction between HOXA11-AS and three candidate genes based on two human predictive databases (IncRNA-miRNA database and miRNAmRNA database). Consequently, 106 unique miRNAs were identified after removing 20 overlapping miRNAs (Figure 6A). Based on the differentially expressed (DE) miRNA profile in keloid fibroblasts with HOXA11-AS knockdown, an expression-based ceRNA network was constructed to screen the downregulated (HOXA11-AS knockdown)- upregulated (candidates among DE miRNAs)-downregulated (SNED1 and NIPAL3) in keloid fibroblasts. Consequently, 51 upregulated miRNAs were identified as competing endogenous miRNAs downstream of HOXA11-AS to downregulate the expression of NIPAL3 
and SNED1 (Figure 6B). Based on both sequence-based and expression-based ceRNA networks, dual validation was conducted to screen 10 upregulated miRNAs as candidate molecular sponges in HOXA11AS-NIPAL3 regulatory pathway (Figure 6C). Based on differentially expressed (DE) profiling in keloid fibroblasts with nonspecific interference, a total of 494 DE miRNAs were identified as nonspecific "noise". After 7 overlapping miRNAs that emerged in 10 candidate miRNAs were removed as noise, three upregulated miRNAs (hsa-miR-19a-3p, hsa-miR-141-3p and hsa-miR-140-5p) were identified as outcome sponging miRNAs to construct a ceRNA network as HOXA11-AS-three upregulated miRNAs (hsa-miR-19a3p, hsa-miR-141-3p and hsa-miR-140-5p)-NIPAL3 in keloid fibroblasts (Figure 6D).

\section{Supplementary Files}

This is a list of supplementary files associated with this preprint. Click to download.

- SupplementaryTableS1.docx

- Supplementarydocument1DEprofiles.rar 